\title{
Digitalização 3D de Ossos Humanos para Ferramentas de Estudo em Anatomia
}

\section{D Digitization of Human Bones for Anatomy Study Tools}

PRIM, Gabriel de Souza; Mestre; Universidade Federal de Santa Catarina gabrielsprim@gmail.com

LEAL, Gabriela Zanella; Graduanda; Universidade Federal de Santa Catarina valkeera26@gmail.com

ROMEIRO, Nicolas C.; Graduando; Universidade Federal de Santa Catarina nicolas.sagaz@gmail.com

CAMPOS, Daniel V.; Graduando; Universidade Federal de Santa Catarina danmpos@gmail.com VIEIRA, Milton Luiz Horn; Professor Doutor Eng.; Universidade Federal de Santa Catarina milton@cce.ufsc.br

\section{Resumo}

Diante da inserção de novas tecnologias em ensino e entretenimento nos últimos anos, discute-se como a tecnologia têm permitido que objetos encontrados no mundo virtual se assemelham muito com os objetos reais. Este trabalho aborda um método capaz de criar um objeto virtual tridimensional verossimilhante com o objeto real criado por meio de um scanner 3D e um fluxo de trabalho em diferentes softwares para criar um objeto que possa ser utilizado em diferentes aplicações, como vídeos de animação e jogos digitais.

Palavras Chave: Digitalização 3D; Ensino; Anatomia; Osteologia.

\begin{abstract}
With the introduction of new technologies in teaching and entertainment in recent years, we discuss how technology have enabled objects found in the virtual world are very similar to the real objects. This paper discusses a method able to create a three-dimensional virtual object verossimilhante with the actual object created using a 3D scanner and a workflow in different software to create an object that can be used in different applications such as animated videos and digital games.
\end{abstract}

Keywords: 3D scanning; Teaching; Anatomy; Osteology. 


\section{Introdução}

O contexto do estudo de anatomia é, de modo geral, inerente a interação do estudante com peças cadavéricas encontradas em anatômicos. Geralmente encontradas em universidades, tratamse de instalações apropriadas para o armazenamento de peças cadavéricas destinadas ao ensino e pesquisa dos ossos e suas relações entre si. Considerando o alto custo e as dificuldades legais na manutenção de um anatômico, não são todos os cursos superiores de medicina que dispõe de um centro de estudos de anatomia devido a dificuldades por apresentar alto custo de manutenção devido ao seus insumos (como o formol e a glicerina) e dificuldades pelos fatores éticos e uma legislação rígida.

Neste contexto, este artigo aborda um método de digitalização de ossos humanos em alta resolução com a finalidade de serem empregados em ferramentas digitais de estudo em anatomia baseado em game engine. Tais ferramentas podem ser utilizadas para auxiliar no ensino/aprendizagem da anatomia óssea diminuindo a dependência da utilização de uma peça real.

O método inicia pela aquisição de dados com a tecnologia de escaneamento 3D.

\section{Tecnologias de Escaneamento 3D e Equipamento Utilizado}

Existem diversos tipos de scanners tridimensionais conhecidos, com vantagens e desvantagens para cada tipo de aplicação. Em linhas gerais, um scanner 3D pode ser classificado como scanners com contato e scanners sem contato. Os scanner com contato se caracterizam como scanner com contato físico com o objeto, com alta precisão demandando maior tempo de aquisição de dados. Os scanner sem contato físico demandam menor tempo para aquisição de dados, com menor precisão quando comparado com os scanners sem contato (PERES, 2013).

A digitalização 3D funciona, em geral, por meio de uma varredura medindo a localização dos pontos no espaço (X,Y e Z), denominada de nuvens de pontos. Um scanner 3D, geralmente, trabalha com a técnica de triangulação na qual se movimenta no plano $X$ e $Y$ através de comandos numéricos (CNC) e, depois de finalizada a varredura do objeto a ser digitalizado, é obtido o mapeamento ponto a ponto da superfície. Assim, é gerada a malha tridimensional que é convertida em um modelo 3D. Os digitalizadores tridimensionais tradicionalmente utilizados no mercado são por sistema a laser, por luz branca ou por fotogrametria (BRENDLER et al., 2016).

A tecnologia de digitalização a laser consiste em usar lasers para projetar no objeto uma ou mais listras finas de feixes de luz e, simultaneamente, os sensores de luz registram o objeto. Aplicando regras geométricas simples, a superfície é medida. A unidade de scanner a laser, que é composta pelo laser, pelo sistema óptico e pelo sensor de luz, é movida ao redor do objeto para digitalizar a superfície (D'APUZZO, 2007).

A tecnologia para o escaneamento baseada na projeção de luz branca (geralmente na forma de listras), é semelhante ao método à laser, porém, em vez de mover a unidade do scanner, luzes são projetadas no objeto e um sensor de luz (por exemplo, uma câmera digital) adquire a imagem. Sistemas mais complexos utilizam dois ou três sensores de luz. As listras na superfície são medidas de forma singular, por meio de triangulação (CHROMY; ZALUD, 2014). 
Por fim, a digitalização por fotometria utiliza técnicas de processamento e modelagem de imagens para a digitalização do objeto. A informação 3D é extraída de imagens 2D.

O scanner 3D utilizado na pesquisa é um sistema de medição ótica. O scanner ATOS Compact Scan 5M, da empresa GOM presente em mais de 45 países. Com tecnologia chamada Blue light, o sistema apresenta baixa sensibilidade a iluminação do ambiente e permite uma área de medição de $40 \mathrm{~mm}^{2}$ até $1200 \mathrm{~mm}^{2}$. A resolução máxima (distância mínima entre dois pontos de escaneamento, sendo quanto menor melhor) é de $0.017 \mathrm{~mm}$ com câmera de 5MP.

Figura 1 - scanner ATOS Compact Scan 5M.

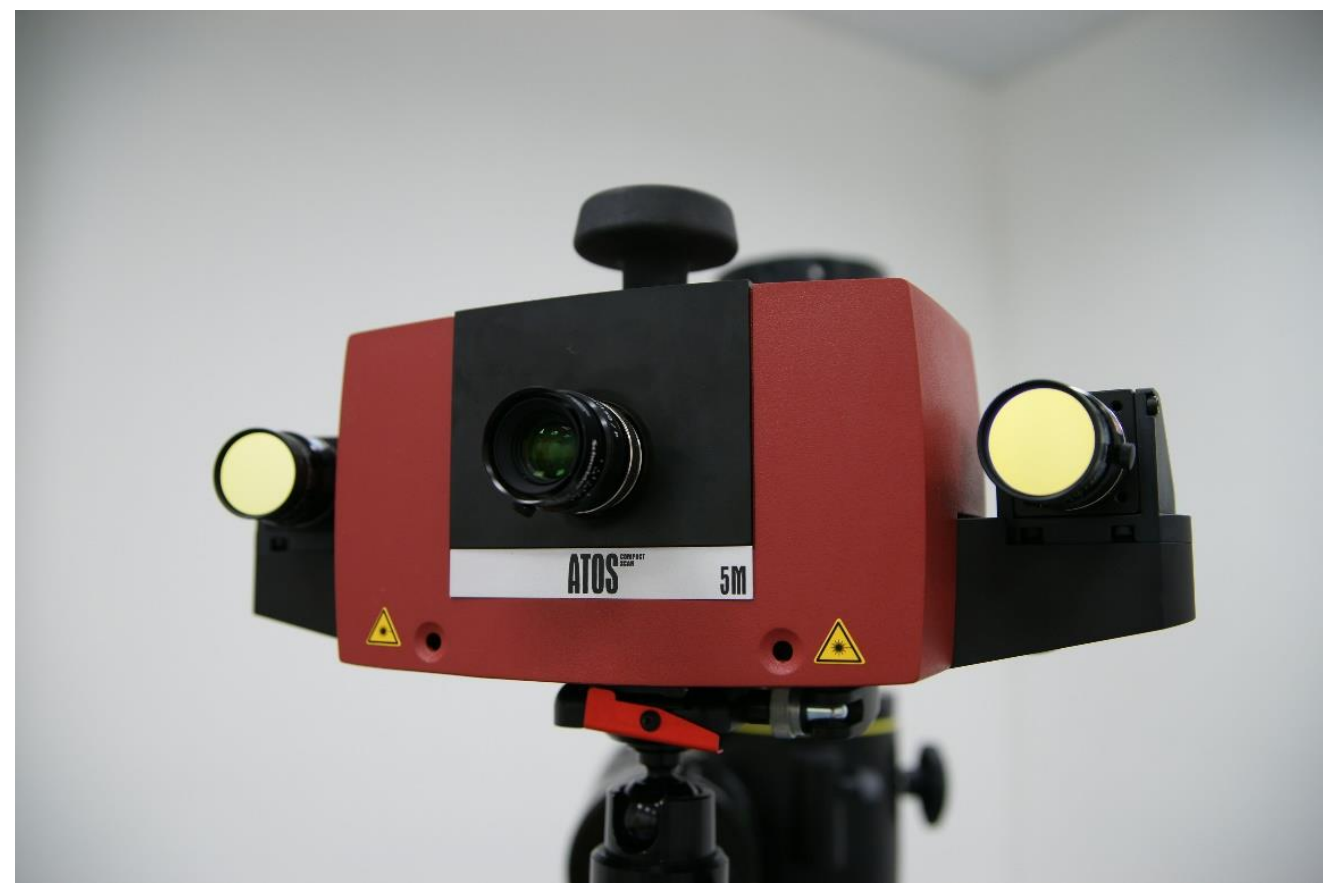

Fonte: o autor.

O equipamento disponível possui dois conjuntos de lentes intercambiáveis: um conjunto de lentes MV600, que possui uma área de captura de $600 \mathrm{~mm} \times 600 \mathrm{~mm}$, utilizado em conjunto com adesivos de $3 \mathrm{~mm}$ dedicado a um trabalho mais ágil e para grandes volumes, e um conjunto de lentes de MV150, que possui uma área de captura de $150 \mathrm{~mm} \times 150 \mathrm{~mm}$, utilizado em conjunto com adesivos de $0,8 \mathrm{~mm}$ dedicado a uma tarefa minuciosa.

\section{Digitalização dos Ossos}

O procedimento de digitalização 3D dos ossos seguiu de acordo com as etapas apontadas por (BRENTON et al., 2007) no contexto da digitalização por imagens de tomografia computadorizada. Adaptando as etapas adotadas ao contexto do Scanner 3D por luz, temos: digitalização do objeto real (aquisição de dados obtidos por tecnologia de escaneamento 3d), refinamento (alteração de características não-necessárias ou prejudiciais para obtenção da qualidade desejada no produto final), classificação e render da superfície (computação das fontes bidimensionais em volumes tridimensionais para visualização no formato de imagens). 


\subsection{Digitalização do Objeto Real}

Os ossos foram trazidos do anatômico da Universidade Federal para o laboratório DesignLab para escaneamento 3D. Os ossos foram escolhidos dentre os ossos disponíveis na sala de estudos Figura 2, a partir de seu estado de conservação.

Figura 2 - Estante no Anatômico da Universidade Federal de Santa Catarina de onde foram selecionados as peças para digitalização.

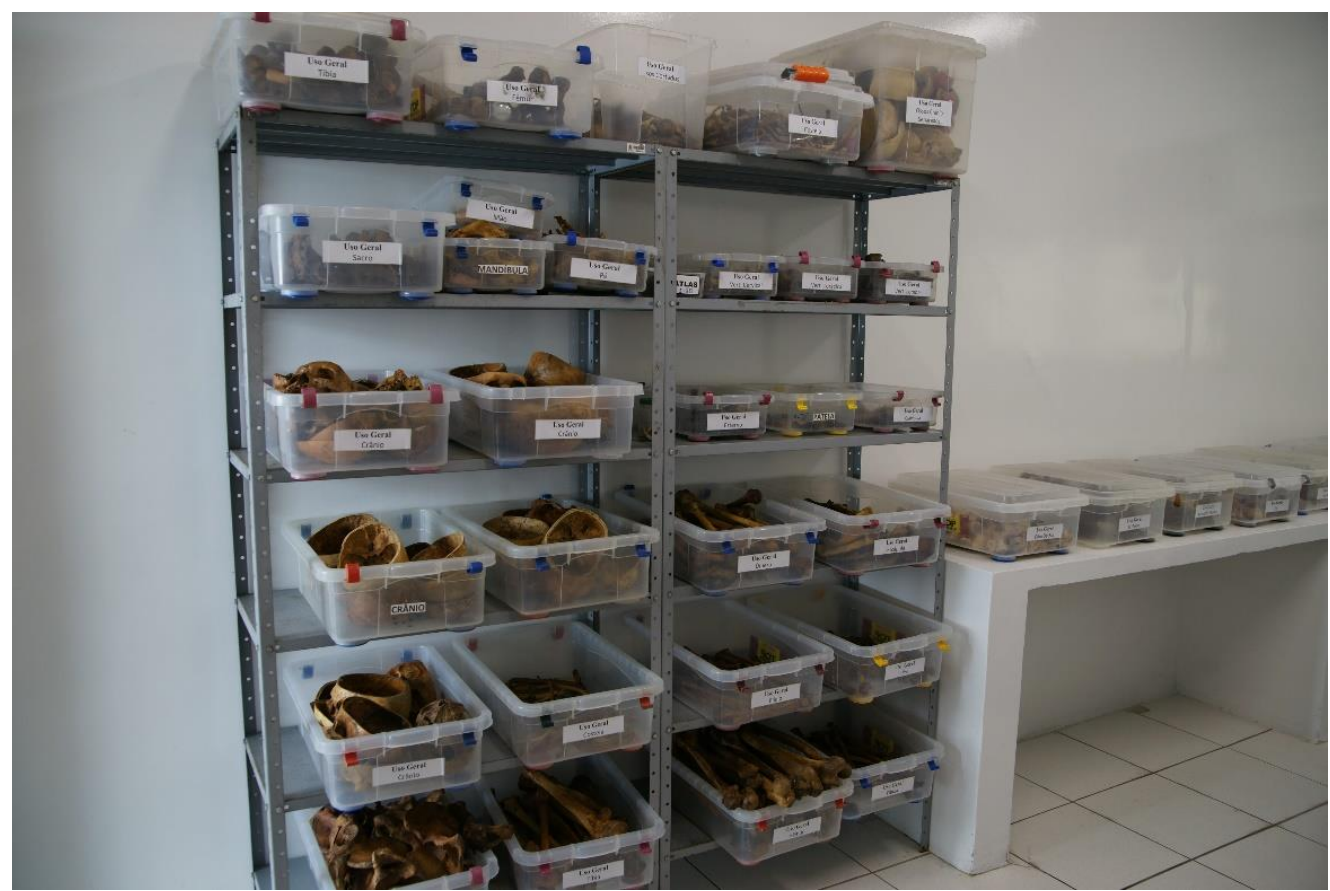

Fonte: o autor.

Considerando a presença de dois conjuntos de lentes disponíveis, testes foram realizados no início do trabalho quando verificou-se que, apesar de tornar o processo mais rápido, a digitalização $3 \mathrm{~d}$ das peças utilizando o conjunto de lentes MV600 e adesivos de $3 \mathrm{~mm}$ não oferece a qualidade esperada para o objeto digitalizado, ilustrado na Figura 3. Sendo assim, os ossos foram digitalizados utilizando um conjuntos de lentes MV150 e adesivos 0,8mm. 
Figura 3 - Comparação da digitalização 3D de um fêmur utilizando o conjunto de lentes MV600 e adesivos de 3mm (esquerda) e com o conjunto de lentes MV150 e adesivos de 0,8mm (direita).

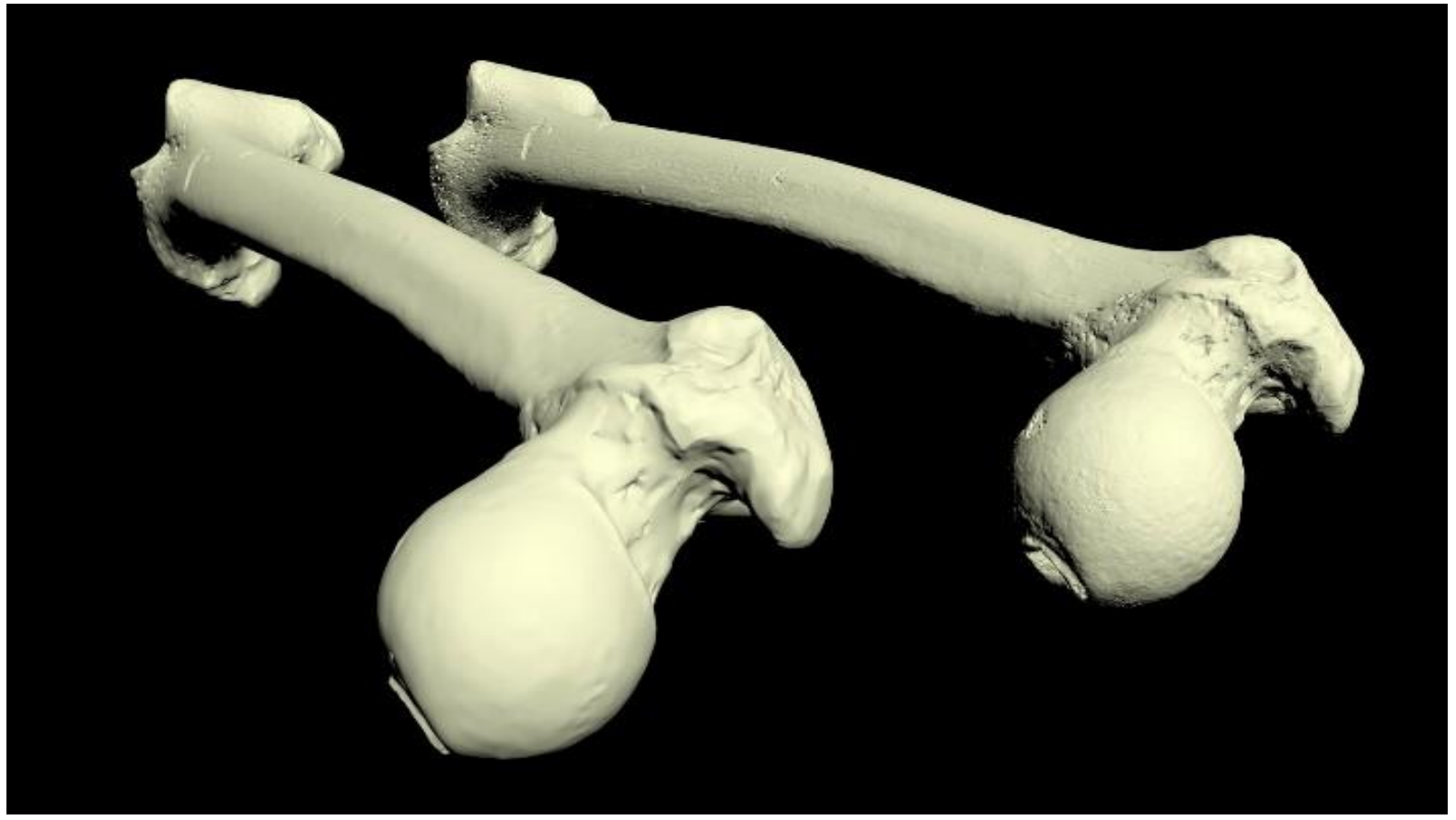

Fonte: o autor.

A maior dificuldade encontrada no processo apareceu durante o processo de digitalização dos ossos menores, como as falanges distais. Devido ao tamanho reduzido e a composição física dos ossos, o scanner 3D não foi capaz de digitalizar essas peças com perfeição. Para solucionar o problema, utilizou-se uma técnica de modelagem 3D onde há uma interferência artística baseada em fotos de alta resolução, obtidas com Câmera Sony Alpha 850 DSLR equipada com lente 70$300 \mathrm{~mm}$ para a menor distorção/perspectiva na imagem, conforme Figura 4. 
Figura 4 - Câmera Sony Alpha 850 DSLR equipada com lente 70-300mm.

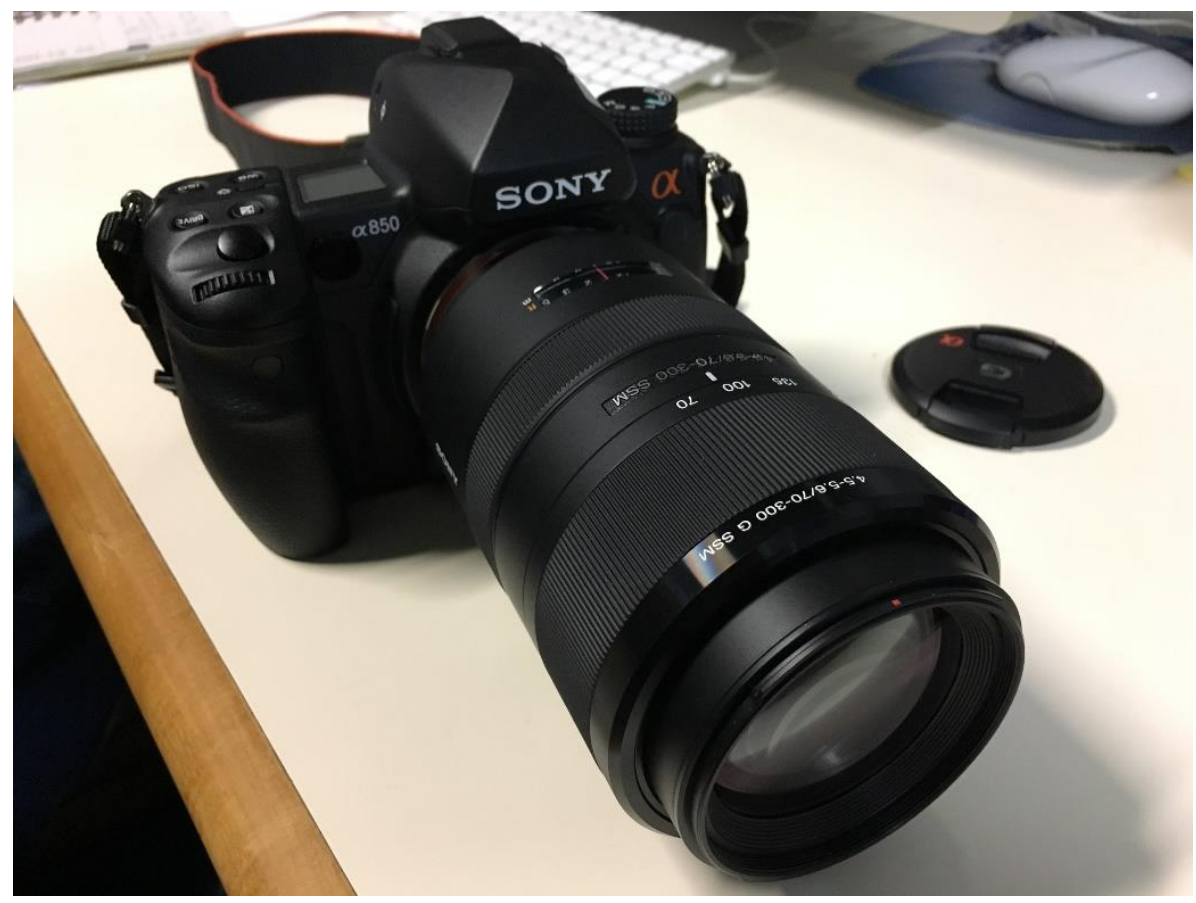

Fonte: o autor.

As imagens foram obtidas de ângulos ortogonais, como exemplificado na Figura 5, e inseridas em softwares da Autodesk 3DS Max e Mudbox para modelagem e preparação para inserção na game engine.

Figura 5 - Fotos obtidas de ângulos ortogonais de uma Falange Distal Il do membro inferior direito.

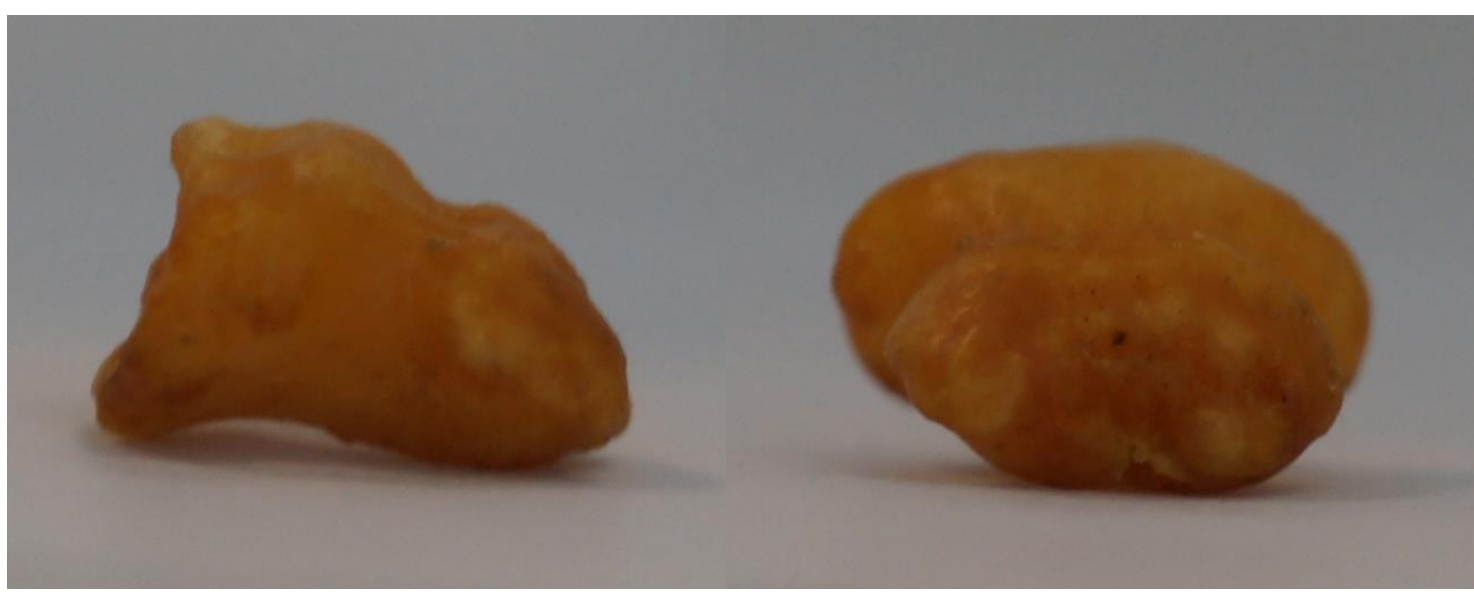

Fonte: o autor.

Para auxiliar no processo de modelagem 3D, utiliza-se um paquímetro para obter as dimensões dos ossos a serem modelados, em seguida organiza-se no software uma caixa de referência mantendo as medidas obtidas (ilustrada na Figura 6). Essa caixa serve como base para fazer a modelagem do osso, tal como para fazer as vistas de referência da modelagem com as fotos obtidas. 
Figura 6 - Caixa de vistas ortogonais de uma falange.

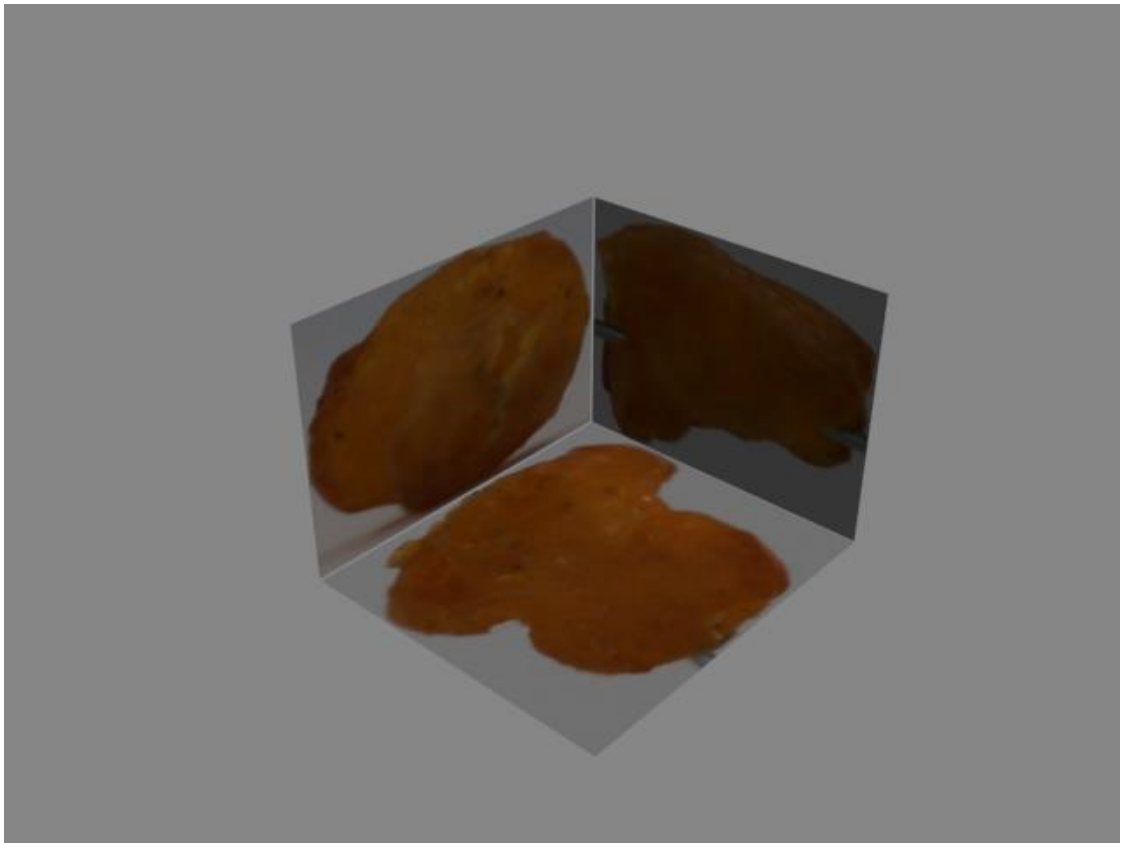

Fonte: o autor.

Através de técnicas básicas de modelagem 3D é montada uma versão da peça com baixa densidade de polígonos, esta é a versão que vai para a visão geral do esqueleto na implementação digital do projeto. Após, utiliza-se de ferramentas do software que aumentam a densidade de polígonos do objeto, como visto na Figura 7, seguida de uma adequação mais refinada da forma da caixa com a forma das fotos de referência.

Figura 7 - Caixa de vistas ortogonais de uma falange.

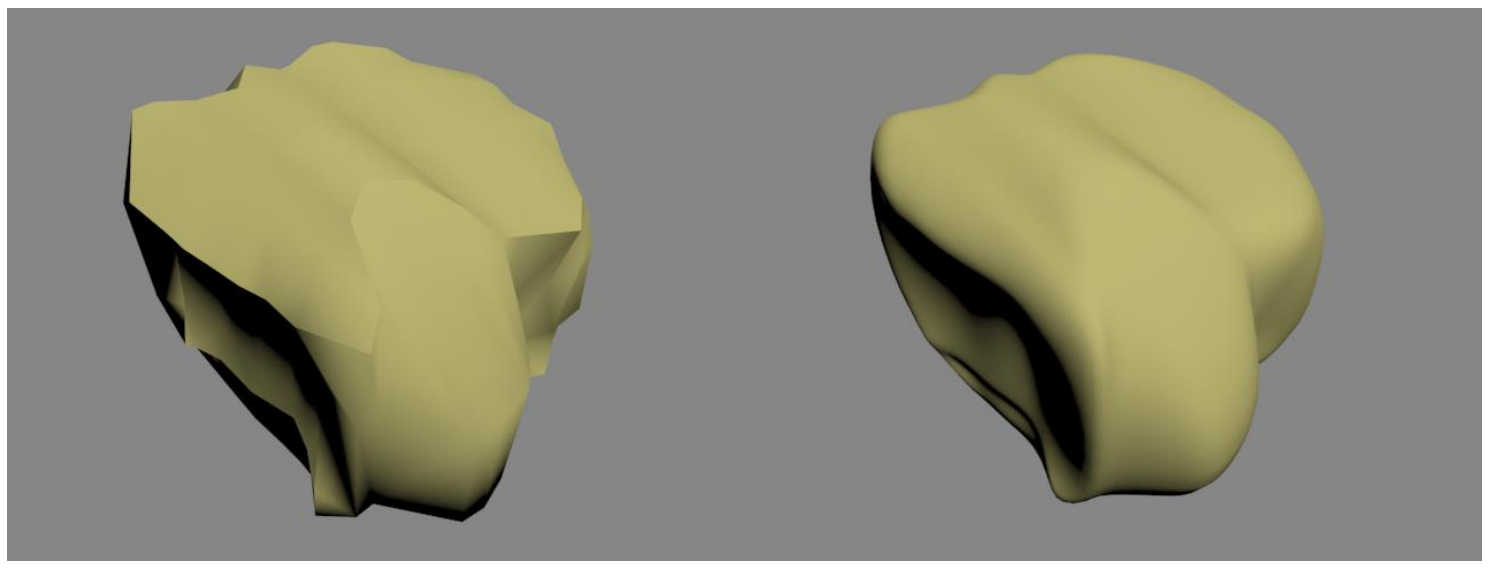

Fonte: o autor.

Por último é aumentada ainda mais a densidade de polígonos da peça e feito um ajuste fino com técnicas de escultura digital conforme apresentado na Figura 8. 
Figura 8 - Peça falange Distal V do Membro Inferior com malha em alta densidade, com texturas e escultura digital aplicadas.

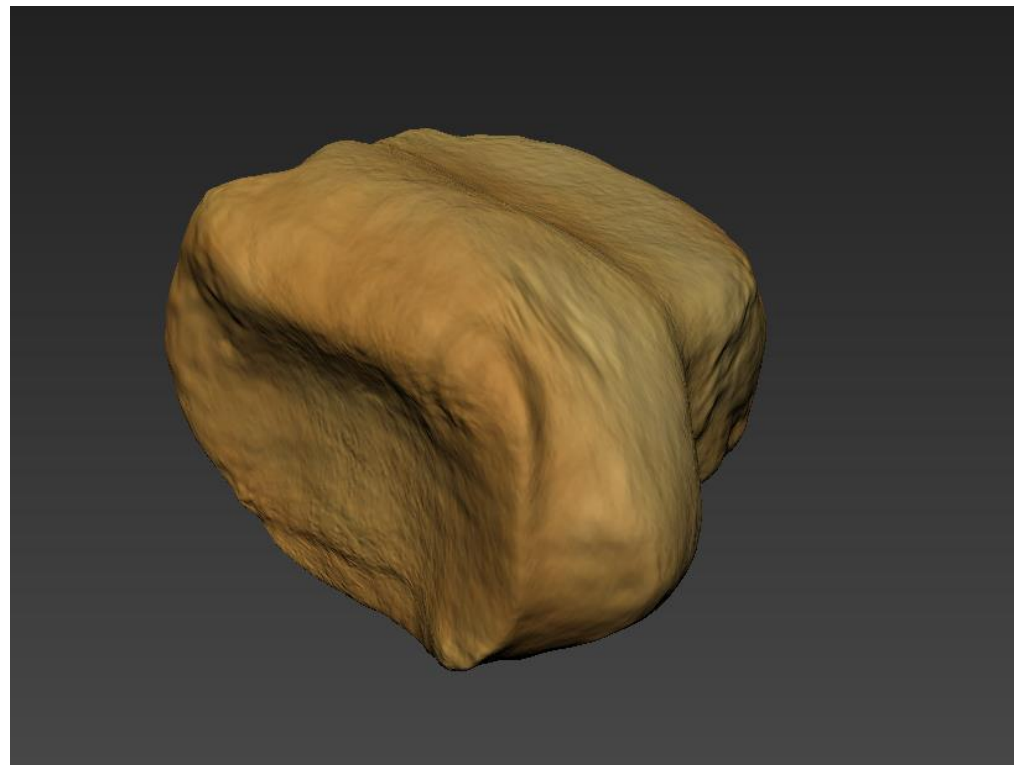

Fonte: $\mathrm{O}$ autor.

Essa versão será usada mais tarde para gerar um mapa de textura à ser aplicado sobre a versão anterior, que por sua vez, será a versão de vista da peça específica na implementação do projeto.

\subsection{Refinamento}

Considerando que a tecnologia utilizada no processo é baseada na luz emitida sobre o objeto, alguns ossos apresentam reentrâncias onde é difícil que a luz seja captada de forma eficaz pelas lentes do equipamento. Sendo assim, algumas pequenas áreas dos ossos requereram interferências artísticas e computacionais para obtenção de toda a área dos ossos.

Ocasionalmente, o escaneamento resulta em malhas com furos, onde a luz não conseguiu ser captada de forma adequada e, consequentemente, deixando falhas no objeto. O programa de escaneamento possui uma ferramenta específica para esse tipo de caso, a ferramenta "Fechar Furos" (Figura 9), essa ferramenta faz cálculos baseados no tamanho dos furos, e também nas posições de bordas mais próximas. Para evitar erros com a ferramenta em questão, as peças reais são conferidas e comparadas com o modelo gerado pelo resultado de escaneamento combinado com o fechamento de furos. 


\section{Artigo Completo}

Figura 9 - Janela da ferramenta "Fechar Furos Automaticamente".

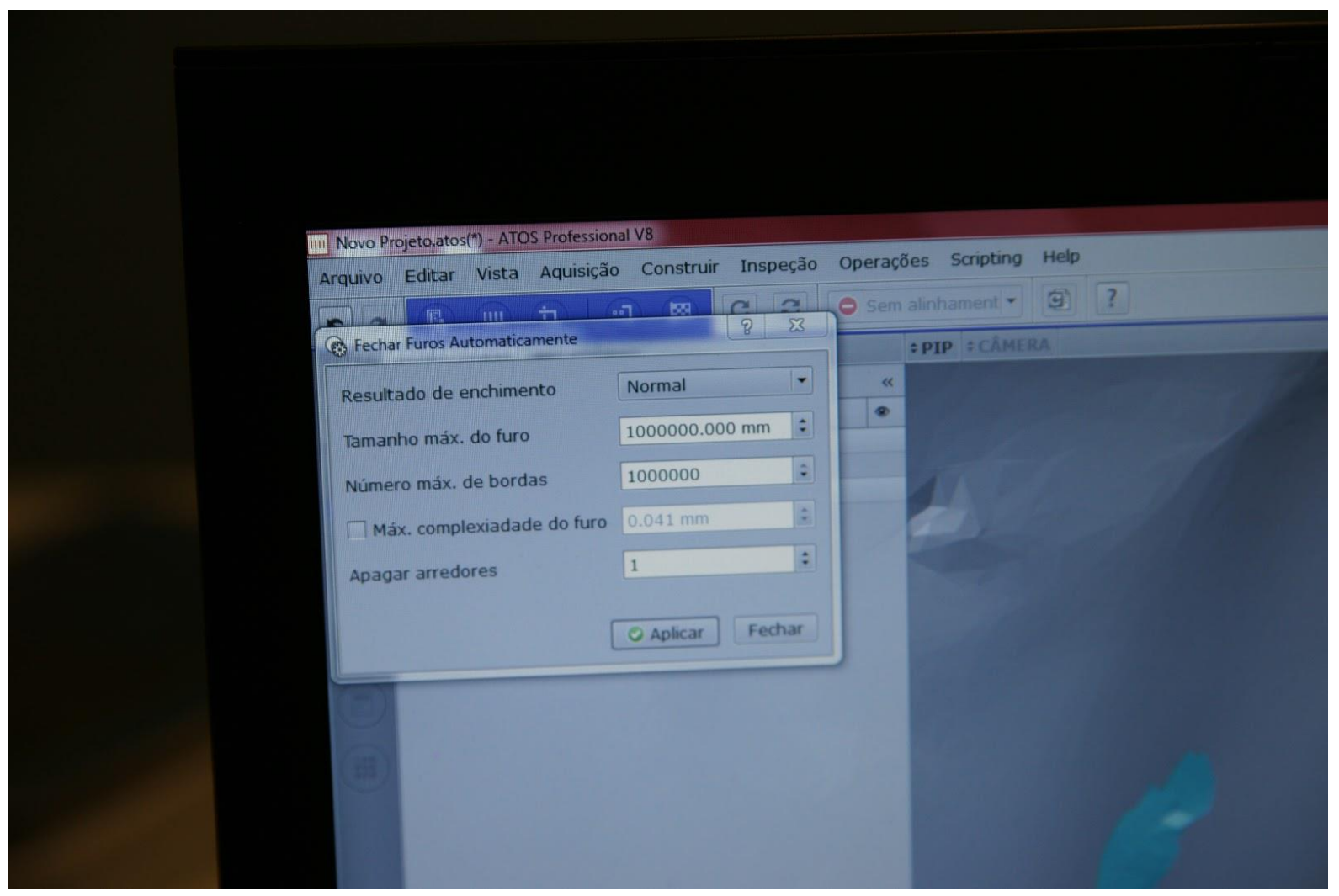

Fonte: O Autor.

Após esse processo, é necessário tratar a malha 3D originada do Scanner, que é exportada em malha de polígonos triangulares. Malhas com polígonos quadriláteros são mais recomendadas para trabalhar na texturização e para uma correta suavização da malha, uma vez que quanto mais densa a malha mais hardware é exigido para o processamento (Andaló, 2015). Para resolver tal problema, é feita a retopologia da malha, refazendo a malha em polígonos quadriláteros. Foi utilizada a ferramenta MudBox para esse fim, observando-se o resultado na Figura 10. 
Figura 10 - Malha original (à esquerda) e Malha com retopologia (à direita).

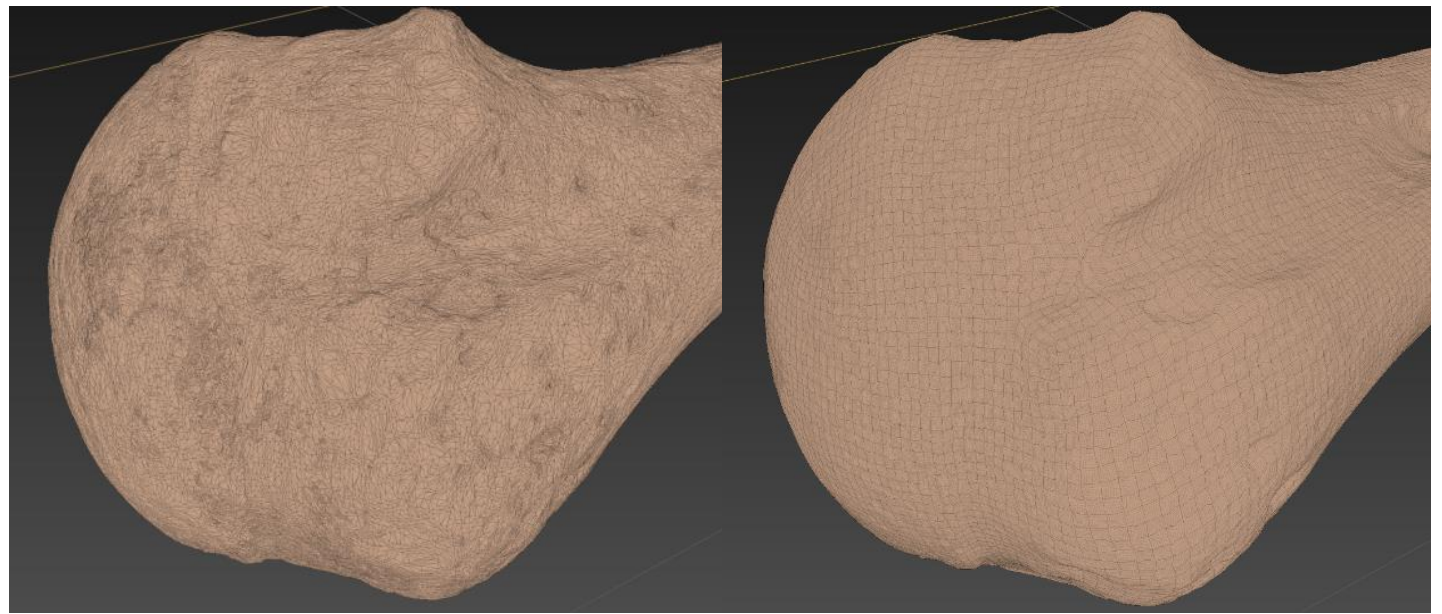

Fonte: $\mathrm{O}$ autor.

Como percebido na figura acima, existe uma perda de detalhes entre a malha original e a retopologia, isso é resolvido criando um mapa normal, apresentado no item 1.1.4.

Após a retopologia ser feita, uma redução da malha é necessária para que se tenha uma malha de baixa densidade.

\subsection{Classificação}

Os ossos foram classificados diante da nomenclatura de Frank H. Netter, por ser o padrão utilizado no curso de medicina da Universidade Federal de Santa Catarina. Dependendo da aplicação, pode-se utilizar nomenclatura adotada por outros autores, mas para fins de desenvolvimento, a classificação de Netter foi adotada.

Os ossos dos membros superior e inferior são digitalizados de apenas um lado (direito ou esquerdo) devidamente registrado. Os ossos do membro oposto são gerados através de "espelhamento".

\subsection{Render da Superfície}

Considerando que o scanner utilizado nesta pesquisa não capta cores, é preciso criar um arquivo de imagem capaz de dar um aspecto natural ao osso. Uma vez que mapeados, os modelos estão prontos para a pintura digital, feita através do software Mudbox, que permite trabalhar diretamente sobre o modelo tridimensional.

Para chegar a um resultado próximo do objeto real, um conjunto de três mapas é feito: mapa difuso, especular e normal. O difuso determina as cores presentes na superfície do modelo; o especular define o quão polido ou reflexivo o material vai aparentar; o mapa normal é gerado a partir de um modelo tridimensional e determina como a luz vai interagir com a superfície, podendo dar a aparência de volumes ou depressões, sem alterar a malha 3D em si. A aplicação destes mapas podem ser observados na figura 11. 
Figura 11 - Peça do fêmur em high poly, da esquerda pra direita, sem aplicação de mapas, com o mapa normal e com os mapas difuso e especular.

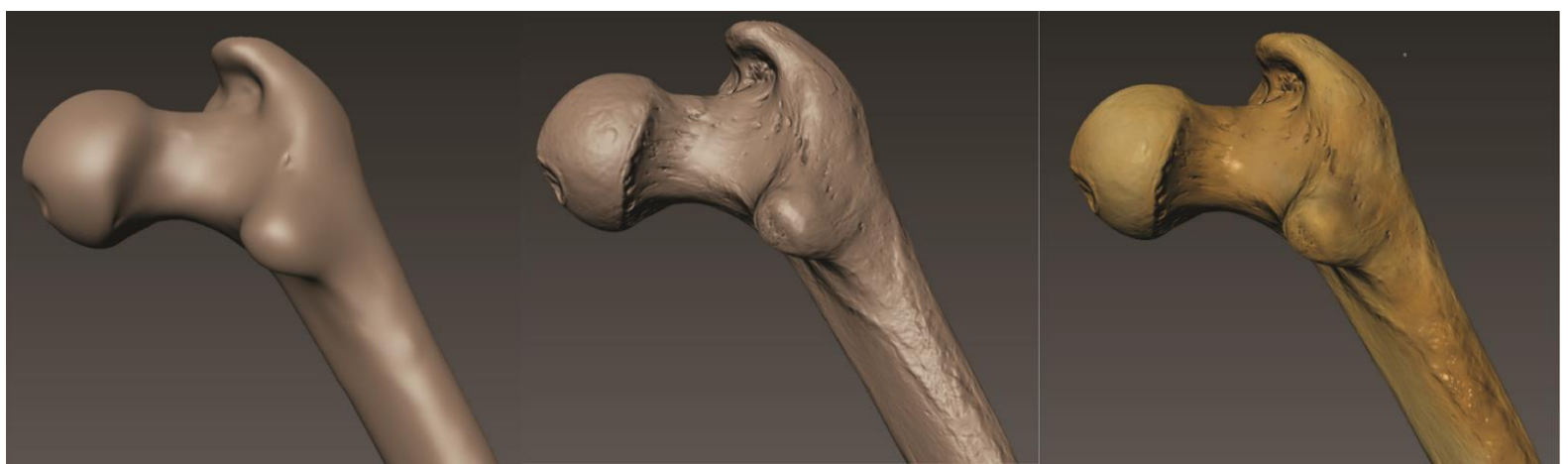

Fonte: $\mathrm{O}$ autor.

Os ossos que foram digitalizados através do scanner têm um total de três modelos: o original retirado do scan, o de alta densidade e o de baixa densidade. Os dois últimos são destinados para utilização em games.

O modelo original é um arquivo com uma complexidade muito alta, tornando sua implementação inviável e por isso é utilizado para a criação de mapa Normal, importante para aprimorar o modelo de alta densidade, oferecendo maior nível de detalhes. O modelo de alta densidade é destinado a visualizações de alta qualidade e é o produto principal deste método. Enquanto o modelo de baixa densidade é destinado a visualizações de baixa qualidade, importante para aplicação em game engines e aplicativos mobile.

No caso dos ossos que devido ao seu tamanho reduzido foram digitalizados através de fotografia e modelagem manual, é gerada apenas a versão de malha de densidade alta e baixa. 0 de densidade mais alta é texturizado, porém na ausência de um modelo do scan, o mapa normal deve ser feito manualmente. Criando uma nova camada de escultura no Mudbox, é possível modelar texturas tridimensionais diretamente na malha, e a partir dela gerar um mapa normal.

Por motivo de diferentes idades e métodos de conservação os ossos tendem a ter colorações bem variadas, variando de tons amarelo-alaranjado, acinzentados ou até marrom escuro. Buscando um padrão aplicável para todos, criou-se uma paleta de cores (figura 10) para ser utilizada na pintura digital dos modelos, inspirada na aparência de um osso recém-extraído, mas com um amarelado leve, para salientar detalhes contidos nos originais. 
Figura 12 - Paleta de cores utilizada para pintura digital.

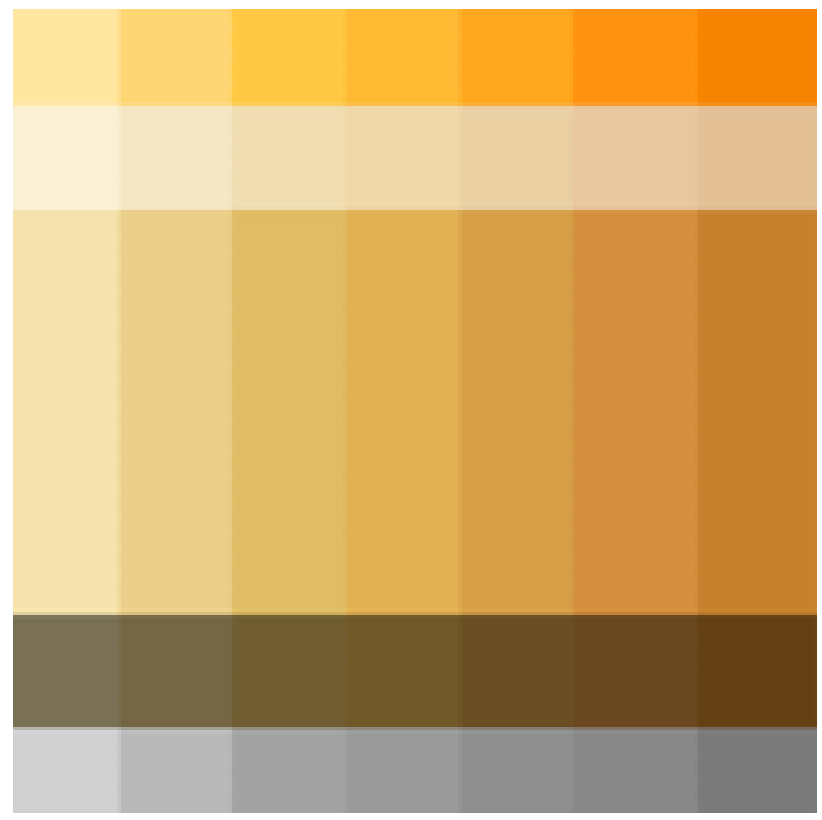

Fonte: $\mathrm{O}$ autor.

No processo de pintura das cores, primeiro é aplicada uma camada de cor clara, predominante no osso, e depois são adicionados detalhes mais escuros com pincéis de variadas texturas, reproduzindo as falhas e pontos com mais desgaste.

O formato escolhido para extrair as imagens foi PNG, devido às características do formato de manter a fidelidade da pintura original. A resolução da imagem varia conforme o tamanho do osso, utilizando 2048 ou 4096 pixels.

\section{Conclusões}

O uso da tecnologia para replicar digitalmente um objeto real e fazê-lo interativo num espaço virtual não é, de forma alguma, uma grande novidade. Todavia, o uso do scanner além de trazer resultados de alta fidelidade e detalhamento, acelerou, de forma geral, o processo de reprodução virtual dos ossos até o momento. Essa reprodução pode ser feita com qualquer tecnologia de escaneamento, mas se faz válido lembrar que a resolução e precisão desta será de grande importância à qualidade final do objeto 3D.

Existem diversos softwares que replicam os métodos utilizadas nesse projeto. É, portanto, possível obter o mesmo tipo de resultado fazendo o uso de outras ferramentas. A escolha dos softwares utilizados foi feita principalmente por questão de domínio e disponibilidade dos autores, podendo ser utilizados outros softwares para efetuar as mesmas tarefas.

Diante do momento atual a inserção das novas tecnologias em educação e entretenimento, 
este trabalho retrata um método capaz de virtualizar um objeto para uma aplicação digital verossimilhante ao objeto real, tornando-se instrumento para a construção de material didático/pedagógico e de entretenimento.

\section{Referências}

ANDALÓ, F. Modelagem e animação 2D e 3D para jogos. Érica: 2015. v. 1, p. 20;22.

ANDRADE, W. M. De. Anatomia mediada por ilustrações para o ensino médico: funções, desenvolvimento, abordagens e tecnologias. [S.I.]: [s.n.], 2015.

BRENDLER, C. F. et al. Uso da digitalização 3D do corpo humano para desenvolvimento de produtos personalizados: Análise comparativa entre os scanners Artec EVA e o Kinect. [S.I.]: [s.n.], 2016. V. 24.

BRENTON, H. et al. Using multimedia and Web3D to enhance anatomy teaching. Computers \& Education, 2007. v. 49, p. 32-53.

CHROMY, A.; ZALUD, L. Robotic 3D scanner as an alternative to standard modalities of medical imaging. SpringerPlus, 2014. v. 3, p. 13.

D'APUZZO, N. Overview of 3D Surface Digitization Technologies in Europe. Image Scanner Optics, 2007.

PERES, F. O. Scanner 3D: Problemas e Soluções. 2013. 